\title{
Nos rôles à titre de pharmaciens d'hôpitaux
}

\author{
par Scot H. Simpson
}

$S^{\mathrm{clo}}$

elon le dictionnaire Larousse, un rôle fait référence à un

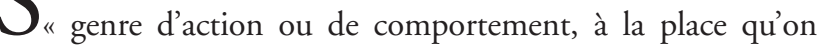
occupe $"^{1}$. Les rôles et les énoncés des rôles servent à donner un contexte et à décrire les exigences en ce qui concerne les devoirs et les responsabilités. Ces énoncés nous aident à comprendre quelles sont les connaissances et compétences que doit posséder la personne qui exerce le rôle. Pris ensemble, les énoncés des rôles précisent les contributions uniques qu'une personne ou qu'un groupe offre à un organisme. La gamme des rôles et des énoncés des rôles peuvent aussi servir à communiquer aux parties prenantes internes et externes ce à quoi elles peuvent s'attendre de cette personne ou de ce groupe. De plus, les énoncés des rôles peuvent servir à mettre en place un ensemble d'attentes sur lequel on s'est entendu et qui permettra d'orienter l'élaboration de politiques et de réaliser des évaluations de performance.

En tant que pharmaciens, notre rôle principal est d'être les experts en pharmacothérapie dans le système de santé-4 ${ }^{2-4}$ Ce rôle nous pousse à nous consacrer aux soins des patients en assurant l'utilisation sécuritaire et efficace des médicaments ${ }^{3}$. Nous pouvons aussi remplir des rôles généraux et agir comme enseignants, porte-paroles, gestionnaires, mentors, collaborateurs et érudits ${ }^{2,4-6}$. La mesure dans laquelle nous serons amenés individuellement à endosser ces rôles supplémentaires dépendra entre autres du milieu de pratique, de l'expérience, des compétences et des champs d'intérêts de chacun. Dans le présent numéro du Journal canadien de la pharmacie hospitalière (JCPH), quatre articles portent sur des exemples de ces rôles supplémentaires.

Dans la rubrique "Recherche originale ", LeBlanc et collab. ${ }^{7}$ se penchent sur leur rôle comme enseignants aidant leurs collègues à apprendre comment surveiller le traitement au moyen d'examens de laboratoire de façon efficace. À titre d'experts en pharmacothérapie, nous, les pharmaciens, devons être en mesure d'utiliser l'ensemble des ressources à notre disposition, ce qui inclut les résultats d'examens de laboratoire, pour ainsi assurer la meilleure pharmacothérapie à un patient. De plus, notre profession embrasse le changement qui l'amène à être plus active dans la prise de décision concernant les médicaments, comme le prouve le nombre de provinces qui ont ajouté l'amorce (ou la prescription) de traitements médicamenteux dans le champ de pratique des pharmaciens ${ }^{8}$. Il est donc essentiel que nous ayons accès aux résultats d'examens de laboratoire ainsi que l'autorisation de prescrire des examens de laboratoire lorsque nécessaire. On voit donc d'un bon œil que bon nombre de provinces sont en voie d'adopter ou sont déjà dotées de politiques permettant aux pharmaciens de prescrire des examens de laboratoire et d'en interpréter les résultats ${ }^{8}$. Cependant, il est aussi important de bien savoir comment se prévaloir de cette compétence; selon LeBlanc et collab. ${ }^{7}$, il s'agit d'une lacune qui doit être comblée par de la formation. Dans leur article, ces auteurs décrivent la réalisation d'un projet pilote sur une intervention éducative dont le but était d'enseigner aux pharmaciens à prescrire des examens de laboratoire pertinents et à en interpréter les résultats lorsqu'ils prennent des décisions concernant la pharmacothérapie.

Dans leur article situé dans la rubrique «Innovations en pratique pharmaceutique ", Stacey et collab. ${ }^{9}$ décrivent leur rôle comme porte-paroles pour une cause : aider à améliorer la pratique de la pharmacie dans un hôpital ougandais. Le savoirfaire des pharmaciens en pharmacothérapie ne devrait pas être arrêtée par des frontières internationales, particulièrement lorsqu'il y a des problèmes d'accès aux médicaments essentiels. En effet, d'importantes organisations, telles que Pharmaciens Sans Frontières - Canada (http://psfcanada.org/), ont été mises sur pied afin d'aider à organiser les réseaux de distribution et des stratégies de gestion des médicaments visant à améliorer la santé des populations des pays en développement. Dans leur article, Stacey et collab. ' décrivent comment ils ont utilisé les déclarations de Bâle sur l'avenir de la pharmacie hospitalière, produites par la Fédération internationale pharmaceutique en $2008^{10}$, comme modèle pour aider l'équipe de direction et le personnel de la pharmacie de l'Hôpital St Mary’s Lacor à réaliser leur vision de la pratique de la pharmacie hospitalière. Ces actions et ces réalisations sont d'excellents exemples des moyens à prendre pour faire la promotion du rôle des pharmaciens dans le développement et la mise en place de politiques et de procédures sur la santé .

Enfin, la chronique "Le pour et le contre » du présent numéro se penche sur le rôle des gestionnaires de pharmacie hospitalière. Elle cherche en particulier à savoir si ces derniers 
devraient ou non continuer de pratiquer en milieu clinique ${ }^{11,12}$. Fait intéressant, notre homologue au sud, l'American Society of Health-System Pharmacists (ASHP), a récemment mis à jour son énoncé sur le rôle et les responsabilités du chef de la pharmacie dans les hôpitaux et dans les systèmes de santét. Dans cet énoncé, l'ASHP souligne l'importance d'accorder une place claire au chef de la pharmacie dans l'organisme pour tirer parti au maximum du savoir-faire de la personne en ce qui touche aux décisions concernant la gestion des médicaments. L'énoncé de l'ASHP décrit différentes exigences du poste (notamment une formation supplémentaire en administration des affaires) et responsabilités (comme la planification stratégique, la supervision du système de gestion des médicaments, la gestion des activités et des ressources humaines du service, et la garantie de la qualité des résultats). Bien que l'énoncé indique aussi que le chef de la pharmacie doit posséder une connaissance approfondie de la thérapeutique clinique, il n'offre aucun conseil précis quant au maintien de la pratique clinique. De part et d'autre, les auteurs du débat «Le pour et le contre " dans le présent numéro du JCPH soulèvent des points importants ${ }^{11,12}$ et je vous invite à lire les arguments respectifs et de discuter du sujet avec vos collègues.

Les rôles et les responsabilités abordés précédemment ne représentent que quelques-uns de ceux qui appartiennent aux pharmaciens. Mais, en plus de ces rôles professionnels, il ne faut pas oublier de souligner l'importance des nombreux rôles que nous devons remplir dans nos vies personnelles auprès de notre famille et de nos amis. Alors que nous amorçons cette nouvelle année, nous devrions prendre un instant pour réfléchir aux rôles de nos vies personnelles et professionnelles, car ils définissent qui nous sommes et ce que les autres attendent de nous.

[Traduction par l'éditeur]

\section{Références}

1. Le dictionnaire Larousse. Les Éditions Larousse; 2016. Publié au : http:// www.larousse.fr/dictionnaires/francais/r\%C3\%B4le/69736?q=role\#68977. Consulté le 27 mai 2016.

2. Model standards of practice for Canadian pharmacists. Ottawa (ON) : Association nationale des organismes de réglementation de la pharmacie; 2009 .
3. Pharmaciens d'hôpitaux : Un document d'information sur l'amélioration de la qualité et de la sécurité d'utilisation des médicaments. Ottawa $(\mathrm{ON})$ : Société canadienne des pharmaciens d'hôpitaux; 2010.

4. Compétences visées par les programmes de formation de premier cycle en pharmacie (Programmes d'entrée dans la profession) au Canada. Edmonton (AB): Association des facultés de pharmacie du Canada; 2010.

5. Miyares MA. The attending pharmacist: roles and responsibilities. Hosp Pharm. 2013;48(9):713-4.

6. ASHP statement on the roles and responsibilities of the pharmacy executive. Am J Health Syst Pharm. 2016;73(5):329-32.

7. LeBlanc JM, Cameron-Coffill K, Symes JL, Kane-Gill S, Duplisea K, Mowatt J. Implementation and assessment of a pharmacy educational program concerning laboratory monitoring for medications. Can J Hosp Pharm. 2017; 70(1):19-26.

8. Champ d'exercice des pharmaciens au Canada. Ottawa $(\mathrm{ON})$ : Association médicale canadienne; 2016. Publié au : www.pharmacists.ca/pharmacy-incanada/scope-of-practice-canada/. Consulté le 23 novembre 2016.

9. Stacey D, Vaillancourt R, Brander L, Chenel N, McMahon E, Wiebe J, et al. Use of the 2008 Basel consensus statements to assess, realign, and monitor pharmacy practice at a tertiary care hospital in northern Uganda: illustrative case study, part 2. Can J Hosp Pharm. 2017;70(1):35-46.

10. The Basel Statements on the future of hospital pharmacy. Am J Health Syst Pharm. 2009;66(5 Suppl 3):S61-6.

11. Beresford L, Dagenais R, Hong J, Louie S. Should institutional pharmacy managers maintain an active clinical practice? Le pour. Can J Hosp Pharm. 2017;70(1):47-8.

12. Leung L, Thorne-Humphrey L, Tkachuk S, Dalen D. Should institutional pharmacy managers maintain an active clinical practice? Le contre. Can J Hosp Pharm. 2017;70(1):49-50.

Scot H Simpson, BBSP, Pharm. D., M. Sc., travaille à la Faculté de pharmacie et des sciences pharmaceutiques de I'Université de l'Alberta, à Edmonton, en Alberta. II est également rédacteur adjoint au Journal canadien de la pharmacie hospitalière.

Intérêts concurrents : Aucun déclaré.

\section{Adresse de correspondance :}

Dr Scot H. Simpson

Faculty of Pharmacy and Pharmaceutical Sciences

3-171 Edmonton Clinic Health Academy

University of Alberta

Edmonton AB T6G 1C9

Courriel : scot@ualberta.ca 